\title{
Nonlinear quantum stochastic resonance
}

\author{
Milena Grifoni and Peter Hänggi \\ Institut für Physik, Universität Augsburg, Memminger Straße 6, D-86135 Augsburg, Germany
}

(Received 23 February 1996)

\begin{abstract}
We investigate quantum stochastic resonance (QSR) in the periodically driven spin-boson system with Ohmic dissipation. For the special case $\alpha=\frac{1}{2}$ of the Ohmic coupling strength we present exact QSR results. For arbitrary Ohmic coupling, the amplitude and the phase of the nonlinear response function vs temperature are studied in a vast regime of temperatures and frequencies. It encompasses a dissipative-dominated incoherent tunneling regime, occurring at "high" temperatures and adiabatic driving, as well as coherent tunneling regimes, reached either at "low" temperatures or/and nonadiabatic frequencies. As a general feature, a principal maximum appears only in parameter regimes where incoherent tunneling dominates over coherent transitions. Moreover, for adiabatic driving, the nonlinear QSR exhibits a noise-induced suppression of higher harmonics accompanied by a characteristic phase change. [S1063-651X(96)05807-2]

PACS number(s): 05.30.- d, 05.40.+j, 33.80.Be, 73.50.Td
\end{abstract}

\section{INTRODUCTION}

The process whereby noise operates on a bistable system enhancing the response to an external periodic signal has been termed stochastic resonance (SR) [1]. While this phenomenon has been the object of many investigations in classical systems [2,3], the role of quantum fluctuations on SR has only started to be explored [4-6]. In particular, the semiclassical SR, that is, SR in the temperature region where quantum corrections to the classical rate of activation are important, has recently been investigated in [4]. On the other hand, the possibility of SR in the deep quantum regime has been addressed in [5], where the role of incoherent tunneling transitions at adiabatic driving frequencies is addressed. In the present work, recent analytical results on the dynamics of the driven spin-boson system will allow us to investigate the nonlinear quantum stochastic resonance (QSR) in a broad parameter range encompassing adiabatic and nonadiabatic frequencies, incoherent and coherent tunneling dynamics. Parts of our analysis have been recently presented in [6].

The investigation of the nonlinear QSR requires one to solve the equation of motion of the nonlinearly driven dissipative bistable system. We shall consider the case of an Ohmic thermal environment characterized by a temperature $T$ and by a dimensionless coupling strength $\alpha$ of the bistable system to the environment.

While the dynamics of the Ohmic dissipative spin-boson system in the absence of driving has been investigated in detail by several authors over the past 15 years (for reviews see [7-9]), that of the driven spin-boson system has only started to be explored [10-15]. In the absence of driving, at sufficiently high temperatures and/or coupling, the dissipative effects are so strong that quantum coherence is completely suppressed by incoherent tunneling transitions (see the upper left inset in Fig. 1). The incoherent dynamics can then be modeled by rate equations. This picture still holds for low-frequency driving [10] [region (a) of Fig. 1]. The role of incoherent transitions at low-frequency driving for classical SR has been addressed in Ref. [16] and its extension to QSR in [5]. In [5] it was found that QSR succeedingly vanishes as the equality between forward and backward transitions is ap- proached. In contrast, for classical and semiclassical SR, the resonance is maximal in the absence of asymmetry [4,17]. As the external frequency $\Omega$ is increased and/or when the temperature is lowered, quantum coherence and/or drivinginduced correlations render the dynamics intrinsically nonMarkovian [13,14] [regions (b) and (c) of Fig. 1]. In this work we investigate both QSR in the linear-response regime, where only thermal equilibrium correlation functions for the spin-boson system need to be evaluated, as well as the nonlinear QSR in the region of the $(T, \Omega)$ plane outlined in Fig. 1 [18]. In particular, for the nonlinear QSR we predict various phenomena, both for coherent (conventional quantum coherent regime for adiabatic driving at low temperatures, driving-induced coherent regime for nonadiabatic driving) and incoherent driven quantum dynamics. Apart from the generic amplification of the first harmonic of the periodic quantum output, we also discover the existence of the quantum analog of noise-induced suppressions (NIS) of higher harmonics [17] and, correspondingly, a characteristic sudden change in the phase shift between periodic response and applied driving signal.

The paper is organized as follows. In Sec. II we describe our model and define the quantities of interest to investigate QSR. As a first example, we consider the case of a weak external signal, where the response function may be evaluated within Kubo's linear-response theory, so that only the knowledge of thermal equilbrium properties of the dissipative two-level system (TLS) is required. We then focus our attention on the linear QSR in the parameter regime $\hbar \Omega \ll k_{B} T$, where a simplified expression for the quantum fluctuation dissipation theorem holds (this restriction is released later on in Sec. IV). The investigation of the linearresponse theory for QSR turns out to be not only interesting per se, but also sheds light on the physics that rules QSR (related to thermal equilbrium properties of the dissipative TLS), together with the qualitative differences between linear and nonlinear QSR.

Sections III-V constitute the main part of this work. There, with the aid of recent results on the dynamics of driven dissipative two-state systems, we investigate the nonlinear QSR. In Sec. III the nonlinear QSR is examined for 


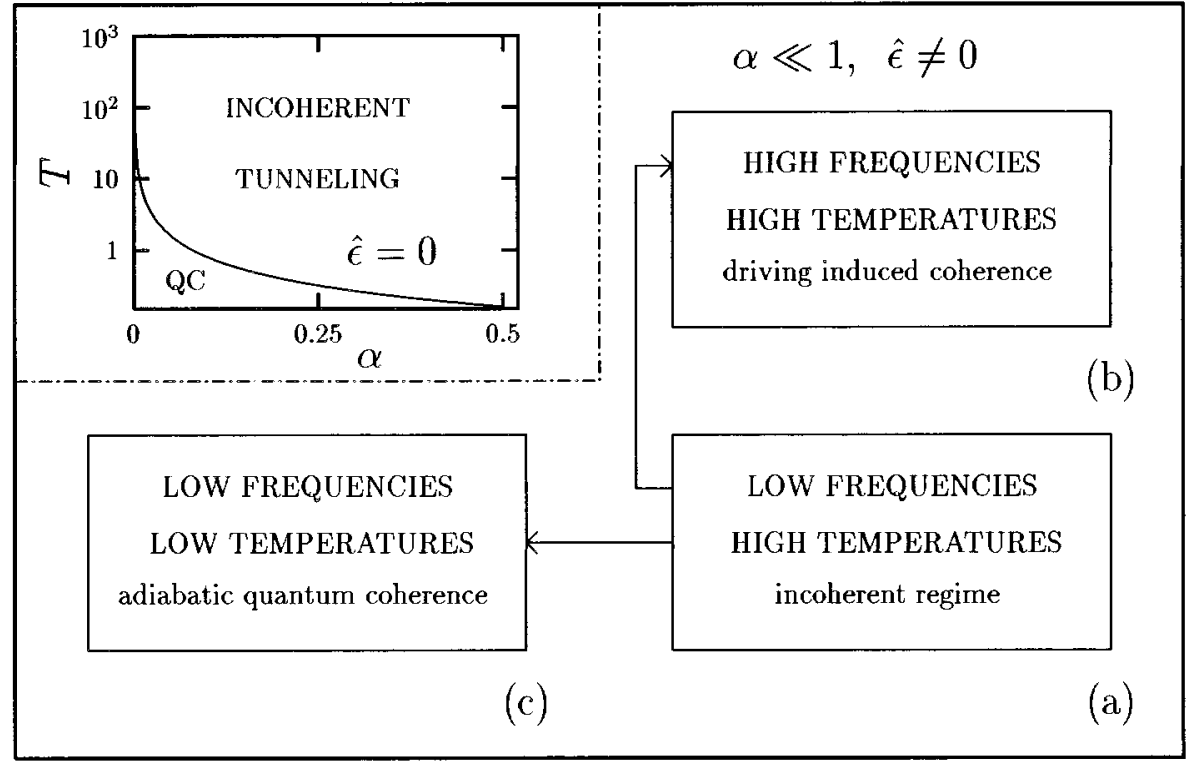

FIG. 1. Dynamics of the driven $(\hat{\boldsymbol{\epsilon}} \neq 0)$ two-state system for weak Ohmic coupling $\alpha$. As the temperature $T$ or frequency $\Omega$ is varied in the $(T, \Omega)$ plane, different tunneling regimes are encountered. For strong coupling, regimes (a) and (b) extend down to the lowest temperatures. For comparison, the static case $(\hat{\boldsymbol{\epsilon}}=0)$ is considered in the inset, where the parameter regions in the $(\alpha, T)$ plane for incoherent or quantum coherent $(\mathrm{QC})$ tunneling are drawn.

the case $\alpha=1 / 2$ of the Ohmic coupling strength. Therein, we obtain exact QSR results. In Sec. IV an iterative scheme is discussed that allows the evaluation of the nonlinear response function, within the non-interacting-blip approximation (NIBA) for the stochastic forces, for any driving strength and frequency. As a first application, we consider the linear-response limit of our results and (within the NIBA) we evaluate the linear susceptibility for any driving frequency $\Omega$. Second, we release the assumption of weak driving strengths and investigate the nonlinear QSR in the regimes of adiabatic driving where (within the NIBA) the dynamics is Markovian and of nonadiabatic driving where a driving-induced coherence occurs. In Sec. V we study the nonlinear QSR in the regime of weak coupling and low temperatures (where coherent tunneling occurs and the NIBA fails). In doing so we use the results of a golden rule approach to the TLS dynamics. Finally, in Sec. VI we present our conclusions.

\section{THE BISTABLE MODEL FOR SR}

As a working model we consider a TLS Hamiltonian. Here the bath is described as an ensemble of harmonic oscillators with a bilinear coupling in the TLS bath coordinates and we allow for an externally applied harmonic force $(\hbar \hat{\epsilon} / a) \cos \Omega t$, of amplitude $\hbar \hat{\epsilon} / a$ and frequency $\Omega$. Then, the driven spin-boson Hamiltonian reads

$$
\begin{aligned}
H= & -\frac{\hbar}{2}\left(\Delta \sigma_{x}+\epsilon_{0} \sigma_{z}\right)-\frac{\hbar \hat{\epsilon}}{2} \cos (\Omega t) \sigma_{z} \\
& +\frac{1}{2} \sum_{\alpha}\left(\frac{p_{\alpha}^{2}}{m_{\alpha}}+m_{\alpha} \omega_{\alpha}^{2} x_{\alpha}^{2}-c_{\alpha} x_{\alpha} a \sigma_{z}\right) .
\end{aligned}
$$

The $\sigma$ 's are Pauli matrices and the eigenstates of $\sigma_{z}$ are the basis states in a localized representation, while $a$ is the tunneling distance. The tunneling splitting energy of the symmetric TLS is given by $\hbar \Delta$, while the asymmetry energy is $\hbar \epsilon_{0}$. Finally, the dissipative influence of the bath is fully characterized by a zero average $\langle\xi(t)\rangle_{0}=0$ and by the correlation function

$$
\langle\xi(t) \xi(0)\rangle_{0}=\frac{\hbar}{\pi} \int_{0}^{\infty} d \omega J(\omega) \frac{\cosh [\omega(\hbar \beta / 2-i t)]}{\sinh (\omega \hbar \beta / 2)}
$$

of the stochastic force $\xi=\Sigma_{\alpha} c_{\alpha} x_{\alpha}[7,8]$. Here $\beta=1 / k_{B} T$ is the inverse of the temperature and \langle\rangle$_{0}$ denotes the ensemble average with respect to the bath Hamiltonian with all the coupling constants $c_{\alpha}$ set to zero. $J(\omega)$ is the spectral density of the environment and we make the specific choice of Ohmic dissipation $J(\omega)=\left(2 \pi \hbar / a^{2}\right) \alpha \omega e^{-\omega / \omega_{c}}$, where $\alpha$ is the dimensionless coupling strength and $\omega_{c}$ is a cutoff frequency [7-9]. In the following we shall assume that the cutoff frequency $\omega_{c}$ in the environment modes is the highest frequency of the problem.

Finally, the dynamical quantity $P(t)=\left\langle\sigma_{z}(t)\right\rangle$ is considered to be the output of the system to the external perturbation and the averaged power spectrum $\bar{S}(\omega)$

$$
\bar{S}(\omega)=\int_{-\infty}^{+\infty} d \tau e^{i \omega \tau} \bar{C}(\tau)
$$

defined as the Fourier transform of the averaged correlation function $\bar{C}(\tau)$

$$
\bar{C}(\tau)=\frac{\Omega}{2 \pi} \int_{0}^{2 \pi / \Omega} d t \frac{1}{2}\left\langle\sigma_{z}(t+\tau) \sigma_{z}(t)+\sigma_{z}(t) \sigma_{z}(t+\tau)\right\rangle,
$$

is the quantity of interest to investigate SR [20]. The combined influence of dissipative and driving forces at intermediate times results in a reduction of the coherent tunneling motion of the isolated TLS by incoherent tunneling transitions. For times $t$ large compared to the time scale of the transient dynamics, the motion acquires the periodicity of the external perturbation and $P(t)$ and $\bar{C}(\tau)$ reach the asymptotic values 


$$
\begin{aligned}
\lim _{t \rightarrow \infty} P(t) & =P_{\mathrm{as}}(t)=\sum_{m=-\infty}^{\infty} P_{m}(\Omega, \hat{\epsilon}) e^{-i m \Omega t} \\
& =\sum_{m=-\infty}^{\infty}\left|P_{m}(\Omega, \hat{\epsilon})\right| e^{-i\left(m \Omega t-\varphi_{m}\right)}
\end{aligned}
$$

and

$$
\lim _{\tau \rightarrow \infty} \bar{C}(\tau)=C_{\text {as }}(\tau)=\sum_{m=-\infty}^{\infty}\left|P_{m}(\Omega, \hat{\epsilon})\right|^{2} e^{-i m \Omega \tau} .
$$

The harmonics $P_{m}$ of $P(t)$ present the quantity of interest to investigate the nonlinear QSR. The amplitudes $\left|P_{m}\right|$ determine the weights of the $\delta$ spikes of the averaged spectral power density in the asymptotic state $S_{\text {as }}(\omega)$ via the relation $[6,19]$

$$
S_{\text {as }}(\omega)=2 \pi \sum_{m=-\infty}^{\infty}\left|P_{m}(\Omega, \hat{\epsilon})\right|^{2} \delta(\omega-m \Omega) .
$$

In order to investigate QSR we shall examine the scaled power amplitude $\eta_{m}$ in the $m$ th frequency component of $S_{\text {as }}(\omega)$ and the associated phase shift $\varphi_{m}$ as well, i.e.,

$$
\begin{gathered}
\eta_{m}(\Omega, \hat{\boldsymbol{\epsilon}})=4 \pi\left|P_{m}(\Omega, \hat{\boldsymbol{\epsilon}}) / \hbar \hat{\boldsymbol{\epsilon}}\right|^{2}, \\
\varphi_{m}(\Omega, \hat{\boldsymbol{\epsilon}})=\arctan \left(\frac{\operatorname{Im} P_{m}(\Omega, \hat{\boldsymbol{\epsilon}})}{\operatorname{Re} P_{m}(\Omega, \hat{\boldsymbol{\epsilon}})}\right) .
\end{gathered}
$$

As a final remark we observe that, for weak external signals, only the fundamental power amplitude $\eta_{1}$ and phase shift $\varphi_{1}$ are different from zero to linear order in the external strength. Within such an approximation, these quantities are related to the amplitude and phase of the linear susceptibility, respectively. Hence they can be also evaluated by computing correlation functions at thermal equilibrium. The results of this procedure are discussed in the following subsection for the case of slow driving fields $\hbar \beta \Omega \ll 1$.

For strong driving forces, the linear-response approximation breaks down and the evaluation of $\eta_{1}$ and $\varphi_{1}$, or of higher harmonics, involves the computation of statistical quantities of the full Hamiltonian (2.1). As we shall see, in the nonlinear regime strong nonlinear effects such as a noiseinduced suppression of higher-order harmonics may appear.

\section{Linear response for QSR}

For weak external signals, $P_{\mathrm{as}}(t)$ can be evaluated within the linear-response approximation. Then, only the harmonics $0, \pm 1$ of $P_{\text {as }}(t)$ in Eq. (2.5) are different from zero. In particular, $P_{0}$ becomes just the thermal equilibrium value $P_{\text {eq }}$ of the operator $\sigma_{z}$ in the absence of driving and $P_{ \pm 1}=\hbar \hat{\epsilon} \widetilde{\chi}( \pm \Omega)$ is related by Kubo's formula [21] to the linear susceptiblility $\widetilde{\chi}_{q q}(\Omega)=a^{2} \widetilde{\chi}(\Omega)$ for the particle position $q=a / 2 \sigma_{z}$, where

$$
\tilde{\chi}_{q q}(\Omega)=\frac{-i}{\hbar} \int_{-\infty}^{+\infty} d \tau e^{-i \Omega \tau} \theta(\tau)\langle[q(\tau), q(0)]\rangle_{\beta} .
$$

Here $\theta(\tau)$ is the Heaviside function and [,] denotes the commutator and \langle\rangle$_{\beta}$ the thermal statistical average of the full system in the absence of the external periodic force $(\hat{\boldsymbol{\epsilon}}=0)$. Hence, from the definitions of Eq. (2.8), the study of the power amplitude $\eta_{1}$ or of the phase $\varphi_{1}$ reduces to the evaluation of the linear susceptibility, yielding

$$
\begin{gathered}
\eta_{1}(\Omega)=4 \pi|\widetilde{\chi}(\Omega)|^{2}, \\
\varphi_{1}=\arctan \left(\frac{\operatorname{Im} \widetilde{\chi}(\Omega)}{\operatorname{Re} \widetilde{\chi}(\Omega)}\right) .
\end{gathered}
$$

Further, the absorptive part $\operatorname{Im} \widetilde{\chi}(\Omega)$ of the susceptibility is related to the Fourier transform $\widetilde{C}(\Omega)$ of the symmetrized correlation function $C(\tau)=C_{q q}(\tau) / a^{2}$, where $C_{q q}(\tau)$ $=\frac{1}{2}\langle q(t+\tau) q(t)+q(t) q(t+\tau)\rangle_{\beta}$, by the fluctuation dissipation theorem [22]

$$
\hbar \operatorname{Im} \widetilde{\chi}(\Omega)=\tanh (\hbar \beta \Omega / 2) \widetilde{C}(\Omega) .
$$

The real part $\operatorname{Re} \widetilde{\chi}(\Omega)$ follows from Eq. (2.12) through the Kramers-Kronig relations. Hence the study of the linear SR, within an approach based on Kubo's theory, involves the evaluation of correlation functions in thermal equilibrium. The computation of the symmetrized correlation function $\widetilde{C}(\Omega)$ can be carried out approximately $[7,8,23,24]$. As we shall see, an investigation of the linear response for QSR will be fundamental to understand some general characteristics of QSR, together with the qualitative differences between the linear and nonlinear QSR.

Let us first consider the case of weak coupling $\alpha \ll 1$, which exhibits the richest behavior as the temperature is varied. At low temperatures the tunneling dynamics is dominated by quantum coherent effects even in the presence of dissipation [see the inset in Fig. 1, where QC denotes the parameter region in the $(\alpha, T)$ plane where quantum coherence occurs [7-9]]. To be definite, at low temperatures $k_{B} T<E / 2 \pi \alpha$ [where $E:=\hbar\left(\Delta_{e}^{2}+\epsilon_{0}^{2}\right)^{1 / 2}$ denotes the bath renormalized energy difference between the two energy levels and $\Delta_{e}(\alpha)$ the renormalized tunneling splitting] and weak coupling, the two-level system undergoes damped coherent oscillations of frequency $E / \hbar$ and lifetime $\gamma_{\text {coh }}$. Superimposed to this is an incoherent tunneling motion with decay time $\gamma_{\text {rel }}$ towards the thermal equilibrium value $P_{\text {eq }}=\hbar \epsilon_{0} / E \tanh \left(E / 2 k_{B} T\right)$. The corresponding symmetrized correlation function $\widetilde{C}(\Omega)$ has been evaluated in [23]. It exhibits two narrow resonances of width $\gamma_{\text {coh }}$ around $\Omega= \pm E / \hbar$ related to the damped coherent oscillations of the TLS, and a quasielastic peak of width $\gamma_{\text {rel }}$ at $\Omega=0$, describing incoherent relaxation towards thermal equilibrium. Within a Lorentzian approximation valid at $\hbar \beta \Omega \ll 1$, the linear susceptibility $\widetilde{\chi}(\Omega)$ is readily obtained by use of the fluctuation dissipation theorem Eq. (2.12) and reads

$$
\widetilde{\chi}(\Omega)=\widetilde{\chi}_{\mathrm{coh}}(\Omega)+\widetilde{\chi}_{\mathrm{rel}}(\Omega),
$$

where 


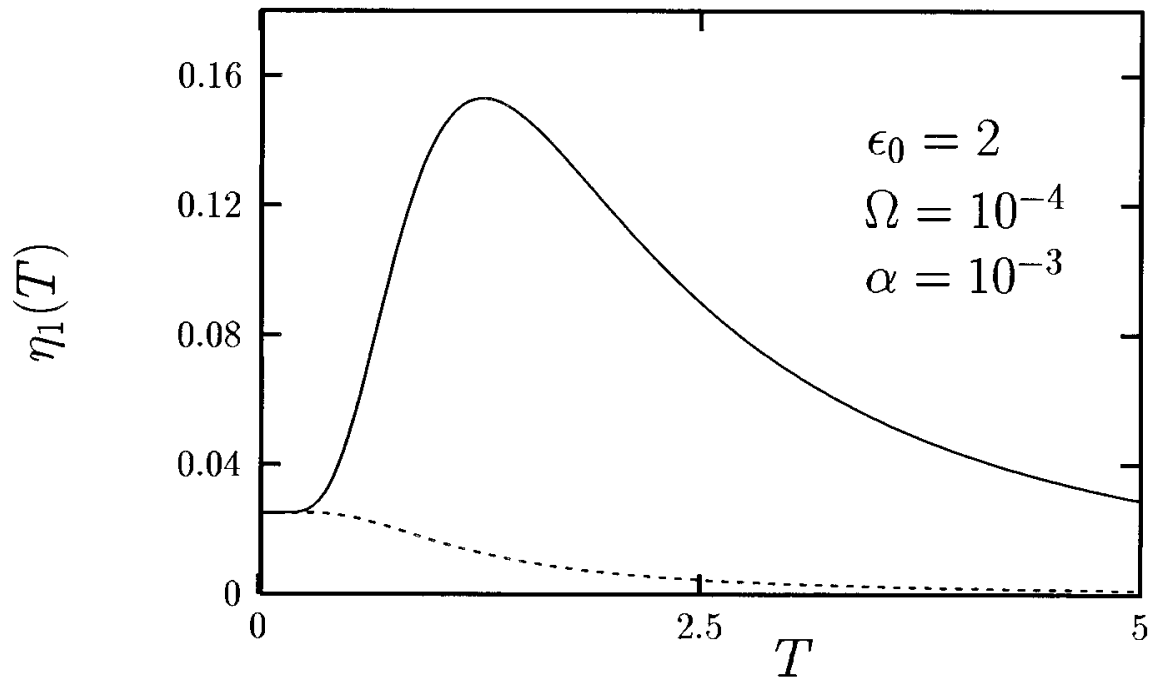

FIG. 2. Amplification vs temperature of the fundamental amplitude $\eta_{1}$ for weak signals in the region of quantum coherent tunneling (full line). Because the coherent contribution ( 2.14) to $\eta_{1}$ decreases monotonically (dashed line), the QSR maximum arises solely because of incoherent transitions. Here and in the following figures, frequencies are in units of $\Delta_{e}$ and temperatures in units of $\hbar \Delta_{e} / k_{B}$

$$
\begin{aligned}
\tilde{\chi}_{\mathrm{coh}}(\Omega)= & \frac{\hbar \Delta_{e}^{2}}{4 E^{2}} \tanh \frac{E}{2 k_{B} T} \\
& \times\left(\frac{1}{E / \hbar+\left(\Omega+i \gamma_{\mathrm{coh}}\right)}+\frac{1}{E / \hbar-\left(\Omega+i \gamma_{\mathrm{coh}}\right)}\right), \\
\tilde{\chi}_{\mathrm{rel}}(\Omega)= & \left(\frac{\hbar \epsilon_{0}}{E}\right)^{2} \frac{1}{4 k_{B} T} \frac{1}{\cosh ^{2}\left(E / 2 k_{B} T\right)} \frac{1}{1-i \Omega \gamma_{\mathrm{rel}}^{-1}},
\end{aligned}
$$

and the dissipation renormalized tunneling matrix $\Delta_{e}$ is

$$
\Delta_{e}=\Delta\left(\frac{\Delta}{\omega_{c}}\right)^{\alpha /(1-\alpha)}[\cos (\pi \alpha) \Gamma(1-2 \alpha)]^{1 /(2-2 \alpha)} .
$$

Finally, the rates $\gamma_{\mathrm{coh}}$ and $\gamma_{\text {rel }}$ are given by

$$
\begin{aligned}
& \gamma_{\mathrm{rel}}=\pi \alpha\left(\frac{\hbar \Delta_{e}}{E}\right)^{2} \frac{E}{\hbar} \operatorname{coth} \frac{E}{2 k_{B} T}, \\
& \gamma_{\mathrm{coh}}=\frac{\gamma_{\mathrm{rel}}}{2}+2 \pi \alpha\left(\frac{\hbar \epsilon_{0}}{E}\right)^{2} \frac{k_{B} T}{\hbar} .
\end{aligned}
$$

Hence the linear susceptibility $\widetilde{\chi}(\Omega)$ is the sum of a coherent and of an incoherent contribution, the former being the only one that survives as $T \rightarrow 0$. From Eqs. (2.10)-(2.15) the linear QSR can now be straightforwardly investigated. In Fig. 2 the power amplitude $\eta_{1}$ in $(2.10)$, i.e., the squared amplitude of the linear susceptibility $\widetilde{\chi}$, is plotted versus temperature in the parameter region where quantum coherence occurs (solid line). The frequencies are expressed in units of $\Delta_{e}$ and the temperature is given in units of $\hbar \Delta_{e} / k_{B}$. Because the coherent contribution (2.14) decreases monotonically with temperature (see the dashed line), we find the intriguing result that the maximum for QSR arises because of the incoherent relaxational dynamics of an asymmetric TLS towards thermal equilibrium (with $\epsilon_{0}=0$, one has $\widetilde{\chi}_{\text {rel }}=0$ for a symmetric TLS). Finally, the QSR maximum in $\eta_{1}$, obtained by combining Eq. (2.10) with Eqs. (2.14) and (2.15), is attained roughly when $E \approx k_{B} T$. Because of the smooth temperature dependence of the relaxation rate $\gamma_{\text {rel }}$, the maximum is determined by the competition between the detailed balance factor $1 / \cosh ^{2}\left(E / 2 k_{B} T\right.$ ) (which saturates to the value 1 when $2 k_{B} T \geqslant E$ ) and the algebraic factor $T^{-1}$ stemming from $\widetilde{\chi}_{\text {rel }}(\Omega)$ in Eq. $(2.15)$.

As the temperature is increased dissipation-induced incoherent tunneling transitions become increasingly important, until the coherent tunneling motion is destroyed above the temperature $T^{*}(\alpha)=E / 2 \pi \alpha k_{B}$ [23]. Correspondingly, the three peaks of (2.15) merge into a single quasielastic peak of width $\gamma_{\alpha}$,

$$
\widetilde{\chi}(\Omega)=\frac{1}{4 k_{B} T} \frac{1}{\cosh ^{2}\left(\hbar \epsilon_{0} / 2 k_{B} T\right)} \frac{1}{1-i \Omega \gamma_{\alpha}^{-1}} .
$$

On the other hand, for strong coupling $\alpha \geqslant 1 / 2$ incoherent transitions always dominate the dynamics at any temperature. This parameter regime is sketched in the upper left inset of Fig. 1 (incoherent tunneling). Hence, at high temperatures $T>T^{*}(\alpha)$ for weak coupling, as well as always for strong coupling, the dynamics can be described in terms of a Markovian rate equation for the position's probability

$$
\dot{P}(t)=-\gamma_{\alpha}\left[P(t)-P_{\mathrm{eq}}\right],
$$

with rate $\gamma_{\alpha}$ and towards the equilibrium value $P_{\text {eq }}=\tanh \left(\hbar \epsilon_{0} / 2 k_{B} T\right)$. When $\alpha \geqslant 1$ or/and when $T>T^{*}$ one finds that $\gamma_{\alpha}=\operatorname{Re} \Sigma\left(\epsilon_{0}\right)$ and $P_{\mathrm{eq}}=-\tan (\pi \alpha) \operatorname{Im} \Sigma\left(\epsilon_{0}\right) /$ $\operatorname{Re} \Sigma\left(\epsilon_{0}\right)=\tanh \left(\hbar \epsilon_{0} / 2 k_{B} T\right)$. Here

$\Sigma\left(\epsilon_{0}\right)=\frac{\Delta_{e}}{\pi}\left(\frac{\hbar \beta \Delta_{e}}{2 \pi}\right)^{1-2 \alpha} \frac{\Gamma\left(\alpha+i \hbar \beta \epsilon_{0} / 2 \pi\right)}{\Gamma\left(1-\alpha+i \hbar \beta \epsilon_{0} / 2 \pi\right)}$

and $\Gamma(z)$ denotes the gamma function. Again, from an analysis of Eq. (2.19) the linear QSR in the regime of incoherent tunneling can be investigated. Similar to the previously discussed QC case, the QSR maximum is attained roughly when $\hbar \epsilon_{0} \approx k_{B} T$. The QSR is determined by the competition between the detailed balance factor $1 / \cosh ^{2}\left(\hbar \epsilon_{0} / 2 k_{B} T\right)$, which exponentially increases with the temperature until it saturates to the value 1 when $2 k_{B} T \geqslant \hbar \epsilon_{0}$, and the algebraic 


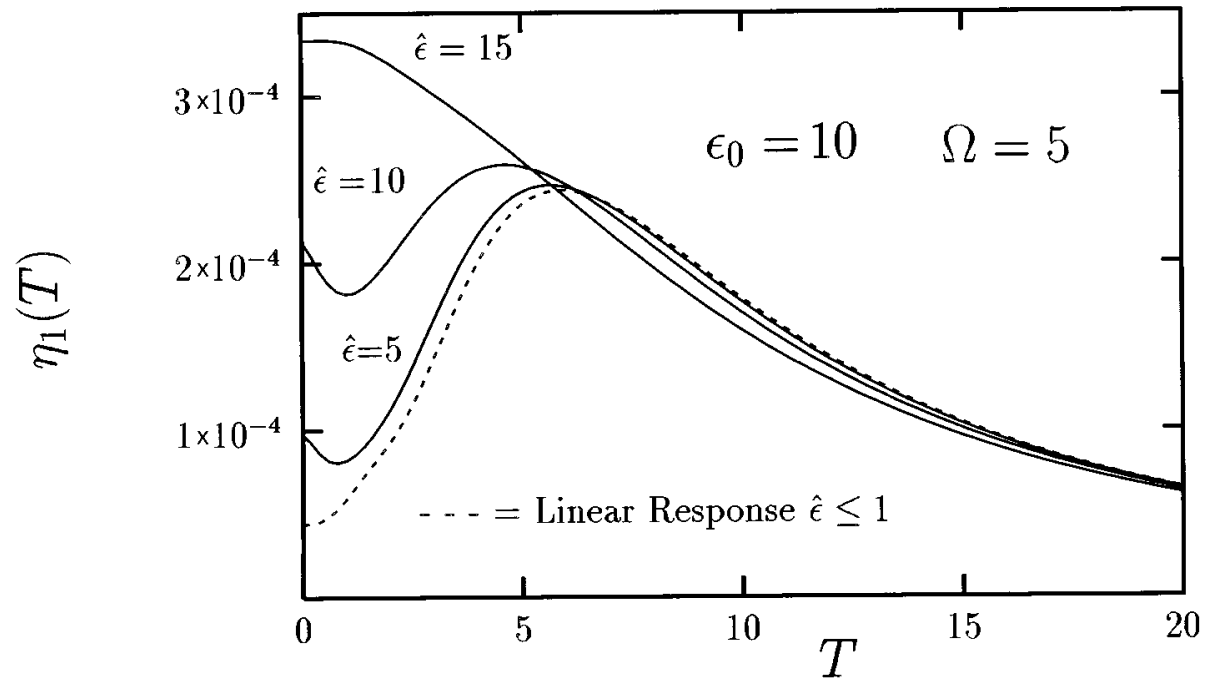

FIG. 3. Amplification vs temperature of the fundamental amplitude $\eta_{1}$ [cf. (2.8)] via quantum $\mathrm{SR}$, for different driving strengths $\hat{\epsilon}$ in the exactly solvable case $\alpha=1 / 2$ of the Ohmic strength. The principal maximum occurs in the temperature region where incoherent tunneling prevails (see Fig. 4).

factor $T^{-1}$ in the incoherent susceptibility [5]. For a quasisymmetric system $\hbar \epsilon_{0} \ll k_{B} T$ the saturation is immediately attained and the linear susceptibility shows only a monotonic decay as the temperature is increased; hence no SR peak occurs.

\section{AN EXACT SOLUTION FOR THE NONLINEAR QSR: THE CASE $\alpha=1 / 2$}

The main theme of the periodically driven spin-boson system is the modification of the coherent tunneling motion of the isolated TLS by the combined influence of the environmental and driving forces. As discussed in the preceding section, the environmental stochastic forces act in reducing the transient coherent tunneling by incoherent processes until, at long times, quantum coherence is completely suppressed. For driven systems the asymptotic dynamics acquires the periodicity of the periodic force, independently of the characteristics of the thermal bath. An exact formal solution for any strength of the external force, which is in the form of a power series in the tunneling transitions, has been obtained using a real-time path-integral approach in $[10,13,14]$. For arbitrary coupling $\alpha$ one has to resort to approximate solutions of the formal solution. In Secs. IV and V we shall discuss controlled approximations that cover the entire range of parameters outlined in Fig. 1. In this section we shall consider the special value $\alpha=1 / 2$ of the Ohmic friction. In contrast to the classical case, where no exact closed analytical solutions are yet available for the nonlinear dynamics, in the quantum case the two-state approximation of the dynamics within an extended bistable potential allows an exact solution for this special value of the Ohmic strength. As shown in [10], the power series in the tunneling transitions can be summed up exactly in analytic form to give

$$
P_{m}(\Omega, \hat{\epsilon})=\frac{\gamma}{\gamma-i m \Omega} \frac{2 \omega_{c}}{\pi} h_{m}(-i m \Omega, \gamma)
$$

$$
\begin{aligned}
h_{2 k}(\lambda, \gamma)= & (-1)^{k} \sin (\pi \alpha) \int_{0}^{\infty} d \tau e^{-\lambda \tau-\gamma \tau / 2-S(\tau)} \\
& \times e^{-i k \Omega \tau} \sin \left(\epsilon_{0} \tau\right) J_{2 k}\left(\frac{2 \hat{\epsilon}}{\Omega} \sin \frac{\Omega \tau}{2}\right), \\
h_{2 k+1}(\lambda, \gamma)= & (-1)^{k} \sin \pi \alpha \int_{0}^{\infty} d \tau e^{-\lambda \tau-\gamma \tau / 2-S(\tau)} \\
& \times e^{-i(k+1 / 2) \Omega \tau} \cos \left(\epsilon_{0} \tau\right) J_{2 k+1}\left(\frac{2 \hat{\epsilon}}{\Omega} \sin \frac{\Omega \tau}{2}\right) .
\end{aligned}
$$

Here $S(\tau)=2 \alpha \ln \left[\hbar \beta \omega_{c} / \pi \sinh (\pi \tau / \hbar \beta)\right], J_{m}(z)$ is a Bessel function of the first kind, and $\gamma=\pi \Delta^{2} / 2 \omega_{c}$ is the dissipation renormalized tunneling frequency $\Delta_{e}$ [see Eq. (2.16)] for $\alpha=1 / 2$. In Fig. 3 the power amplitude $\eta_{1}(\Omega, \hat{\boldsymbol{\epsilon}})$ is plotted as a function of the temperature for different driving strengths $\hat{\boldsymbol{\epsilon}}$ (frequencies are in units of $\gamma$ and temperatures in units of $\hbar \gamma / k_{B}$ ). Following the discussion of Sec. II, $\eta_{1}$ can be interpreted as the squared amplitude of a generalized nonlinear susceptibility $\chi_{\mathrm{NL}}(\Omega, \hat{\epsilon})$. For highly nonlinear driving fields $\hat{\epsilon}>\epsilon_{0}$ the power amplitude decreases monotonically as the temperature increases (uppermost curve). As the driving strength for periodic driving $\hat{\epsilon}$ is decreased, a shallow minimum followed by a maximum appears when the static asymmetry $\epsilon_{0}$ equals, or slightly overcomes, both the external frequency $\Omega$ and strength $\hat{\epsilon}$ (intermediate curves). For even smaller external amplitudes, the nonlinear QSR can be studied within the linear-response theory (dashed curve), where the linear susceptibility for the special case $\alpha=1 / 2$ takes the analytic form 


$$
\begin{aligned}
\phi(\Omega)= & \psi\left(x_{+}\right)-\psi\left(x_{+}-i \frac{\hbar \beta \Omega}{2 \pi}\right)+\psi\left(x_{-}\right) \\
& -\psi\left(x_{-}-i \frac{\hbar \beta \Omega}{2 \pi}\right)
\end{aligned}
$$

and $x_{ \pm}=\frac{1}{2}+\hbar \beta / 2 \pi\left(\gamma / 2 \pm i \epsilon_{0}\right)$. In the linear region the shallow minimum is washed out and only the principal maximum survives. It is now interesting to observe that, because for the undriven case the TLS dynamics for $\alpha=1 / 2$ is always incoherent down to $T=0$, again the principal maximum arises at the temperature $T$ at which the relaxation process towards thermal equilibrium is maximal. On the other hand, the minimum in $\eta_{1}$ appears in the temperature region where driving-induced coherent processes are of importance. This means that the power amplitude $\eta_{1}$ [or the nonlinear susceptibility $\left.\chi_{\mathrm{NL}}(\Omega, \hat{\epsilon})\right]$ plotted versus frequency shows resonances when $\Omega \approx \epsilon_{0} / n(n=1,2, \ldots)$ (full line in Fig. 4). This is a typical nonlinear effect, which has to be compared with the resonances at $E= \pm \hbar \Omega$ that the coherent part (2.14) of the linear susceptibility exhibits in the quantum coherent regime. Correspondingly, the dynamics is intrinsically nonMarkovian. As the temperature is increased, the coherence is increasingly lost (note the behavior of the dot-dashed and dashed lines in Fig. 4).

\section{INCOHERENT AND COHERENT QSR WITHIN THE NIBA}

In the static case $(\hat{\epsilon}=0)$, the major difficulty for an analytic resummation of the series in the TLS tunneling transitions arises from the bath-induced correlations between different transitions. Further, it is known that a perturbative approach in the Ohmic coupling would fail in describing the destruction of the quantum coherent motion induced by the environmental stochastic forces at finite temperatures. In the presence of time-dependent driving the situation is even more intricate, because one also has to take into account the correlations induced by the external field. The bath-induced correlations can be treated within the non-interacting-blip approximation for the stochastic force $[10,13]$, whose range of validity is, in general, different as compared to the undriven case, and it has to be determined self-consistently. A set of coupled equations for the Fourier coefficients $P_{m}$ has been recently derived for any strength and frequency of the driving force [13], yielding the result

$$
P_{0}=\frac{f_{0}(0)}{g_{0}(0)}-\sum_{m \neq 0} \frac{g_{-m}(0)}{g_{0}(0)} P_{m},
$$

and for $m \neq 0$

$$
P_{m}=\frac{i}{m \Omega}\left(f_{m}(-i m \Omega)-\sum_{m^{\prime}} g_{m-m^{\prime}}(-i m \Omega) P_{m^{\prime}}\right) \text {. }
$$

The coefficients $f_{m}$ are defined by Eq. (3.2) via $f_{m}=\Delta^{2} h_{m}(-i m \Omega, 0)$; for the $g_{m}$ one has

$$
\begin{aligned}
g_{2 k}(\lambda)= & (-1)^{k} \Delta^{2} \cos \pi \alpha \int_{0}^{\infty} d \tau e^{-\lambda \tau-S(\tau)} \\
& \times e^{-i k \Omega \tau} \cos \left(\epsilon_{0} \tau\right) J_{2 k}\left(\frac{2 \hat{\epsilon}}{\Omega} \sin \frac{\Omega \tau}{2}\right), \\
g_{2 k+1}(\lambda)= & (-1)^{k+1} \Delta^{2} \cos \pi \alpha \int_{0}^{\infty} d \tau e^{-\lambda \tau-S(\tau)} \\
& \times e^{-i(k+1 / 2) \Omega \tau} \sin \left(\epsilon_{0} \tau\right) J_{2 k+1}\left(\frac{2 \hat{\epsilon}}{\Omega} \sin \frac{\Omega \tau}{2}\right) .
\end{aligned}
$$

We now observe that the coupled ensemble of equations (4.2) can be converted again into the integro-differential equation

$$
\dot{P}_{\text {as }}(t)=\mathcal{F}(t)-\frac{\Omega}{2 \pi} \int_{0}^{2 \pi / \Omega} d t^{\prime} P_{\text {as }}\left(t^{\prime}\right) \mathcal{L}\left(t^{\prime}, t-t^{\prime}\right)
$$

that describes the time evolution within a period. Here

$$
\mathcal{F}(t)=\sum_{n} e^{-i n \Omega t} f_{n}(-i n \Omega)
$$

$$
\mathcal{L}\left(t, t^{\prime}\right)=\sum_{m, n} e^{-i m \Omega t} e^{-i n \Omega t^{\prime}} g_{m}(-i n \Omega) .
$$

This result, within the NIBA, is still exact and explicitly shows that, even at high temperatures and/or strong coupling, the asymptotic driven dynamics is intrinsically nonMarkovian [in contrast to Eq. (2.20) for the undriven case]. In the following we shall first briefly discuss the linear response limit of Eqs. (4.1) and (4.2) valid for any frequency. Subsequently, we shall release the assumption of weak external fields and we shall study the nonlinear QSR in the case of high-frequency and low-frequency driving, respectively, where two different approximations of Eq. (4.4) are discussed. For the weak-coupling case and not too low temperatures (so that the NIBA still applies), they would represent the regions (a) and (b), respectively, of Fig. 1. Region (c) of Fig. 1 is investigated in Sec. V. In the strong-coupling case, only region (a) or (b) may be attained, but not region (c) because adiabatic quantum coherence at strong friction never occurs.

\section{A. Linear response}

On the assumption that the driving force is weak, Eqs. (4.1) and (4.2) can be evaluated by linearizing them in the amplitude $\hat{\epsilon}$ of the time-dependent force. To linear order in $\hat{\boldsymbol{\epsilon}}$, only the coefficients $f_{m}$ and $g_{m}$ with $m=0, \pm 1$ need to be considered. Further, the linearized coefficients $f_{0}^{(0)}, g_{0}^{(0)}$ are of order zero in $\hat{\epsilon}$, while $f_{1}^{(1)}, g_{1}^{(1)}$ are of order $\hat{\epsilon}$. In terms of these linearized coefficients and of the zeroth-order function

$$
v\left(\Omega, \epsilon_{0}\right)=\Delta^{2} \cos \pi \alpha \int_{0}^{\infty} d \tau e^{i \Omega \tau-S(\tau)} \cos \left(\epsilon_{0} \tau\right),
$$




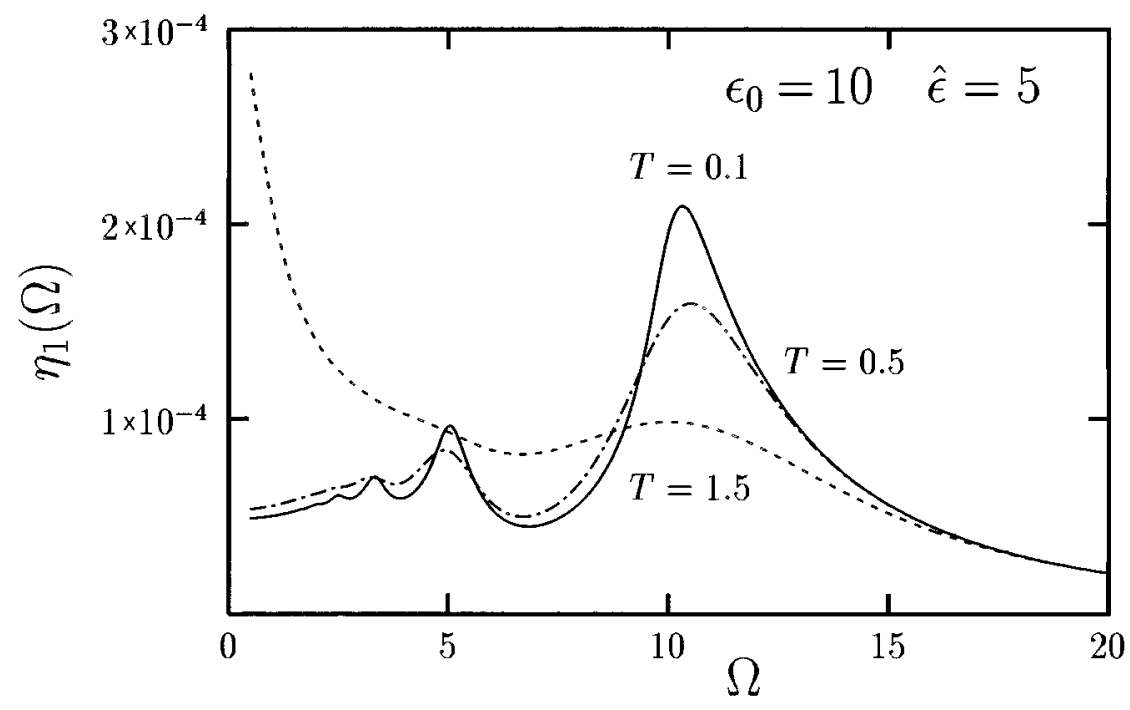

FIG. 4. Fundamental amplitude $\eta_{1}$ vs driving frequency $\Omega$ for different temperatures in the exactly solvable case $\alpha=\frac{1}{2}$. At high temperatures only incoherent relaxation occurs (dashed line). As the temperature is decreased, resonances are found at submultiples $\Omega=\epsilon_{0} / n(n=1,2, \ldots)$ of the static bias (dot-dashed and full lines). These denote the occurrence of driving-induced coherence.

one finds, for the linear susceptibility, the expression

$$
\begin{aligned}
\widetilde{\chi}(\Omega)= & \frac{1}{\hbar \hat{\epsilon}\left[-i \Omega+v\left(\Omega, \epsilon_{0}\right)\right]} \\
& \times\left(f_{1}^{(1)}(-i \Omega)\right. \\
& \left.-g_{1}^{(1)}(-i \Omega) \frac{f_{0}^{(0)}(0)}{g_{0}^{(0)}(0)}\right) .
\end{aligned}
$$

Futher, by comparison with Eq. (2.21) and the definition of $P_{\text {eq }}$ in the line above it, one obtains $f_{0}^{(0)}(0) /$ $g_{0}^{(0)}(0)=P_{\text {eq }}=\tanh \left(\hbar \epsilon_{0} / 2 k_{B} T\right)$ and $g_{0}^{(0)}(0)=\gamma_{\alpha}$. This result, within the NIBA, holds for any driving frequency $\Omega$. It is now interesting to observe that, due to the specific form of the Ohmic interaction $S(\tau)$ [see the line below Eq. (3.2)], whenever the condition $\Omega \ll \alpha k_{B} T / \hbar$ is met, one can linearize the integrands defining $v\left(\Omega, \epsilon_{0}\right)$ and the linearized coefficients $f_{1}^{(1)}(-i \Omega), g_{1}^{(1)}(-i \Omega)$ in the small quantity $\Omega \tau$. We obtain $v\left(\Omega, \epsilon_{0}\right) \simeq v\left(0, \epsilon_{0}\right)=g_{0}^{(0)}(0)$ together with

$$
f_{1}^{(1)}(-i \Omega) \simeq f_{1}^{(1)}(0)=\frac{\hat{\boldsymbol{\epsilon}}}{2} \frac{d}{d \epsilon_{0}} f_{0}^{(0)}(0)
$$

and

$$
g_{1}^{(1)}(-i \Omega) \simeq g_{1}^{(1)}(0)=\frac{\hat{\epsilon}}{2} \frac{d}{d \epsilon_{0}} g_{0}^{(0)}(0)
$$

from which the linear susceptibility is readily found in the simple Lorentzian form of Eq. (2.19). We defer the investigation of QSR in the nonadiabatic regime to the next subsection.

\section{B. High-frequency QSR}

In order to discuss a systematic treatment of Eq. (4.4) useful at high driving frequencies, it is convenient to recast it in the form

$$
\dot{P}_{\mathrm{as}}(t)=\mathcal{F}(t)-\frac{\Omega}{2 \pi} \sum_{m} \mathcal{G}_{m}(t) \int_{0}^{2 \pi / \Omega} d t^{\prime} P_{\mathrm{as}}\left(t^{\prime}\right) e^{-i m \Omega t^{\prime}}
$$

$$
\mathcal{G}_{m}(t)=\sum_{n} e^{-i n \Omega t} g_{m+n}(-i n \Omega)
$$

We observe now that at high frequencies only small- $m$ values in the sum (4.8) are of importance, because they represent contributions almost at resonance with the fast oscillating field. In particular, taking $m=0$ amounts to evaluating $P_{\text {as }}(t)$ to order $1 /\left(\tau_{c} \Omega\right)$. Here $\tau_{c}^{-1}:=\lim _{\hat{\epsilon} \rightarrow 0} g_{0}(0)=\gamma_{\alpha}$ is the only nonvanishing coefficient $g_{m}$ in (4.3) for zero external driving field. It defines the time scale for the system to reach thermal equilibrium in the absence of driving. We recover in this case the high-frequency approximation of (4.2) previously discussed in Ref. [13]. On the other hand, because Eq. (4.8), together with (4.9), within the NIBA is still exact, we can now evaluate an $m$ th harmonic $P_{m}$ of $P_{\text {as }}(t)$ to the order $\left(1 / \tau_{c} \Omega\right)^{s+1}$ if $m \neq 0$, to the order $\left(1 / \Omega \tau_{c}\right)^{s}$ if $m=0$, which we denote $P_{m}^{(s)}$, by use of the zeroth and first $s$ harmonics of $P_{\text {as }}(t)$ by means of the relation

$$
\begin{aligned}
P_{m}^{(s+1)}= & \frac{i}{m \Omega}\left(f_{m}(-i m \Omega)\right. \\
& \left.+\sum_{k=-s}^{s} P_{k}^{(s-|k|)} g_{m-k}(-i m \Omega)\right), \quad m \neq 0,
\end{aligned}
$$

with the initial value $P_{0}^{(0)}=f_{0}(0) / g(0)$. The average value $P_{0}^{(s)}, s \neq 0$, is obtained by inserting Eq. (4.10) in Eq. (4.1).

The dynamics described by (4.10) can be highly coherent, as revealed by a numerical analysis of the Fourier coefficient $P_{1}(\Omega, \hat{\epsilon})$ as a function of the driving frequency: When the external frequency matches fractional values of the intrinsic bias, resonances are found (see the inset in Fig. 5). Finally, we observe that, because a fast oscillating field results in reducing the bath-induced correlations, whenever the NIBA is applicable in the absence of driving, it is justified even better in the presence of a high-frequency driving (i.e., always for strong coupling $\alpha>1$; for weak coupling $\alpha \ll 1$ when $2 \pi \alpha k_{B} T>\hbar \Delta_{e}$ or $\epsilon_{0}>\Delta_{e}$ ). Figure 5 describes the first 


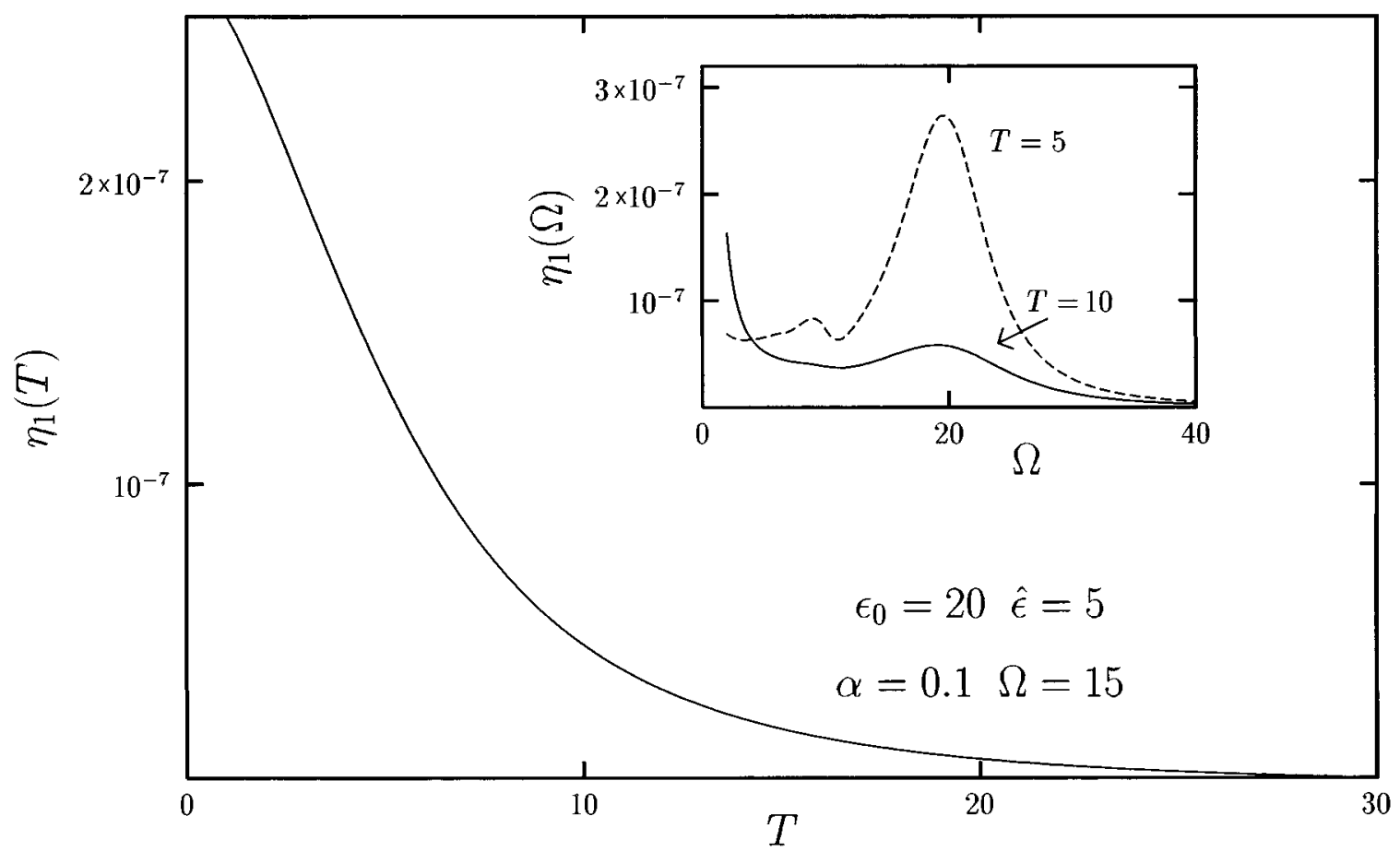

FIG. 5. Monotonic decay vs temperature of the first amplitude $\eta_{1}$ for nonadiabatic driving. As shown in the inset, in the investigated parameter regime the dynamics is dominated by driving-induced coherent transitions. Correspondingly, $\eta_{1}$ does not exhibit a peak as the temperature is varied.

amplitude $\eta_{1}$ versus temperature. In the investigated parameter regime the power amplitude always shows a monotonic decay as the temperature is increased. This is in accordance with the findings of the previous sections (see Figs. 2-4), where the maximum in the power amplitude was found in the temperature regime dominated by incoherent tunneling. This is also in accordance with results on classical SR at high driving frequencies [19].

\section{Low-frequency QSR}

At low frequencies the above discussed truncation scheme is useless because the contribution from all the harmonics has to be considered. Whenever $\Omega \tau_{c} \ll 1$ (that is, $\left.\hbar \Omega \ll 2 \pi \alpha k_{B} T\right)$, we can approximate $g_{m}(-i n \Omega) \simeq g_{m}(0)$ and $f_{m}(-i n \Omega) \simeq f_{m}(0)$ in Eq. (4.5) to get $\mathcal{L}\left(t, t^{\prime}\right) \rightarrow \gamma_{\alpha}(t) \delta\left(t^{\prime}\right)$ and $\mathcal{F}(t) \rightarrow \gamma_{\alpha}(t) P_{\text {eq }}(t)$. Hence $P_{\text {as }}(t)$ in Eq. (4.4) obeys the time-dependent rate equation

$$
\dot{P}_{\mathrm{as}}(t)=-\gamma_{\alpha}(t)\left[P_{\mathrm{as}}(t)-P_{\mathrm{eq}}(t)\right],
$$

with a time-dependent rate $\gamma_{\alpha}(t)=\operatorname{Re} \Sigma[\varepsilon(t)]$ and time-dependent adiabatic equilibrium $P_{\text {eq }}(t)$ $=-\tan (\pi \alpha) \operatorname{Im} \Sigma[\varepsilon(t)] / \operatorname{Re} \Sigma[\varepsilon(t)]$. Here $\Sigma(\epsilon)$ is defined by Eq. (2.21) and $\varepsilon(t)=\epsilon_{0}+\hat{\epsilon} \cos \Omega t$ plays the role of a timedependent adiabatic asymmetry. Finally, the rate equation can be solved in terms of quadratures to give

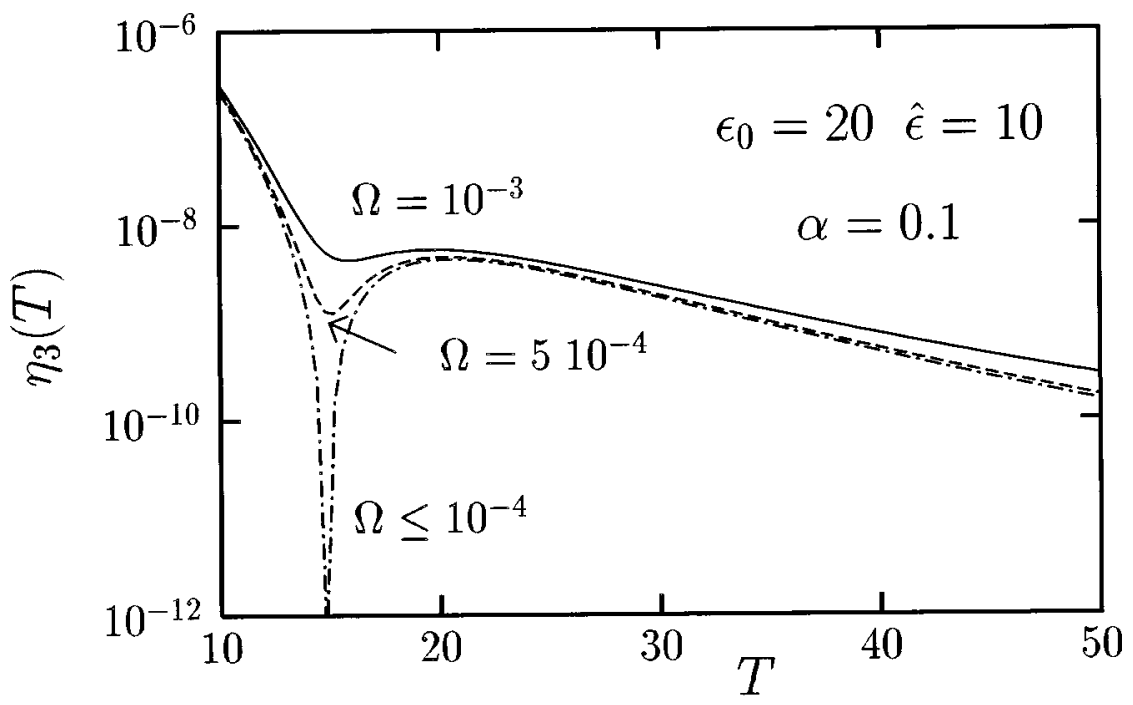

FIG. 6. Noise-induced suppression (NIS) of the third amplitude $\eta_{3}$ vs temperature in the adiabatic incoherent regime (4.12) as the frequency is decreased. 


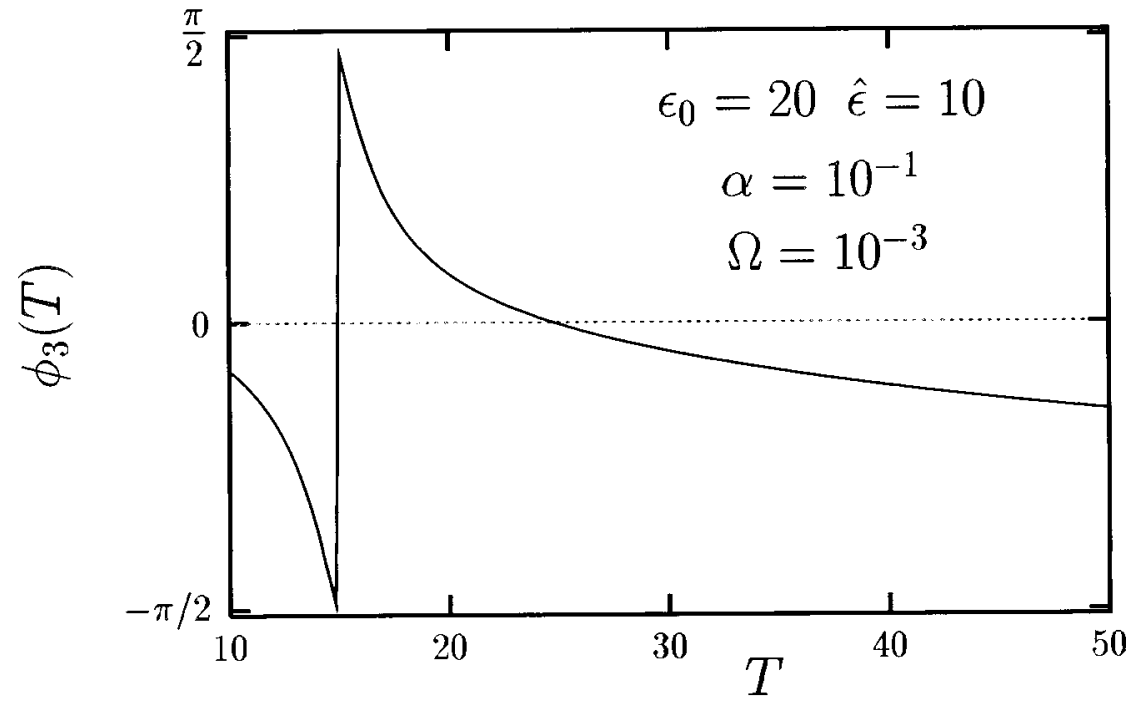

FIG. 7. Discontinuity in the associated phase shift $\varphi_{3}$ in correspondence of the NIS in the amplitude $\eta_{3}$

$$
\begin{aligned}
P_{m}(\Omega, \hat{\boldsymbol{\epsilon}})= & \frac{\Omega}{2 \pi} \int_{0}^{2 \pi / \Omega} d t_{0} \gamma_{\alpha}\left(t_{0}\right) P_{\mathrm{eq}}\left(t_{0}\right) e^{-i m \Omega t_{0}} \int_{0}^{\infty} \mathrm{d} t_{1} \\
& \times e^{-i m \Omega t_{1}} \exp \left(-\int_{t_{0}}^{t_{0}+t_{1}} d t \gamma_{\alpha}(t)\right)
\end{aligned}
$$

Equation (4.12) completely describes the nonlinear SR dynamics at low frequency within the validity range of the NIBA; see also $[10,13]$. We observe that classical nonlinear SR has been recently investigated in a superconducting quantum interference device system [26]. Lowering further the temperature brings the system into the regime of Eq. (4.12), where nonlinear QSR could be observed for a macroscopic quantum system. At low frequencies, the NIBA is justified for strong damping $\alpha>1$, while for weak coupling $\alpha<1$ it is valid in the region $2 \pi \alpha k_{B} T \geqslant \hbar \Delta_{e}$ or $|\varepsilon(t)| \gg \Delta_{e}$. In the small coupling limit $\alpha \ll 1$ the relaxation rate in (4.11) and (4.12) becomes and $P_{\text {eq }}(t)=\tanh \left[\hbar \varepsilon(t) / 2 k_{B} T\right]$. It should be noted that in the nonlinear regime $\hat{\epsilon} \gg \epsilon_{0}$ the presence of the term $2 \pi \alpha k_{B} T$ in the denominator is crucial because $\varepsilon(t)$ is zero at any instant $t$, obeying $t=1 / \Omega \operatorname{arcos}\left(\epsilon_{0} / \hat{\epsilon}\right)$. This explicitly shows the breakdown of any perturbative approach to deal with timedependent problems at high temperatures in the nonlinear regime.

The nonlinear QSR is investigated in Fig. 6, where the third power amplitude $\eta_{3}$ is plotted versus temperature. We find the quantum analog of a NIS of higher harmonics as the frequency is decreased. See Ref. [17] for the phenomenon of NIS in the classical case. As shown in Fig. 7, the related phase shift $\varphi_{3}$ possesses a crossover at the very same value of the temperature at which the NIS appears. To understand the results shown by Figs. 6 and 7 it is convenient to rewrite (4.12) as the sum of a quasistatic $P_{m}^{(\mathrm{qs})}$ and a retarded contribution $P_{m}^{(\text {ret })}$

$$
\gamma_{\alpha}(t)=\pi \alpha \Delta_{e}^{2} \frac{\varepsilon(t)}{(2 \pi \alpha k T)^{2}+\varepsilon(t)^{2}} \operatorname{coth} \frac{\hbar \varepsilon(t)}{2 k_{B} T}
$$

$$
P_{m}(\Omega, \hat{\boldsymbol{\epsilon}})=P_{m}^{(\mathrm{qs})}(\hat{\boldsymbol{\epsilon}})+P_{m}^{(\mathrm{ret})}(\Omega, \hat{\boldsymbol{\epsilon}}),
$$

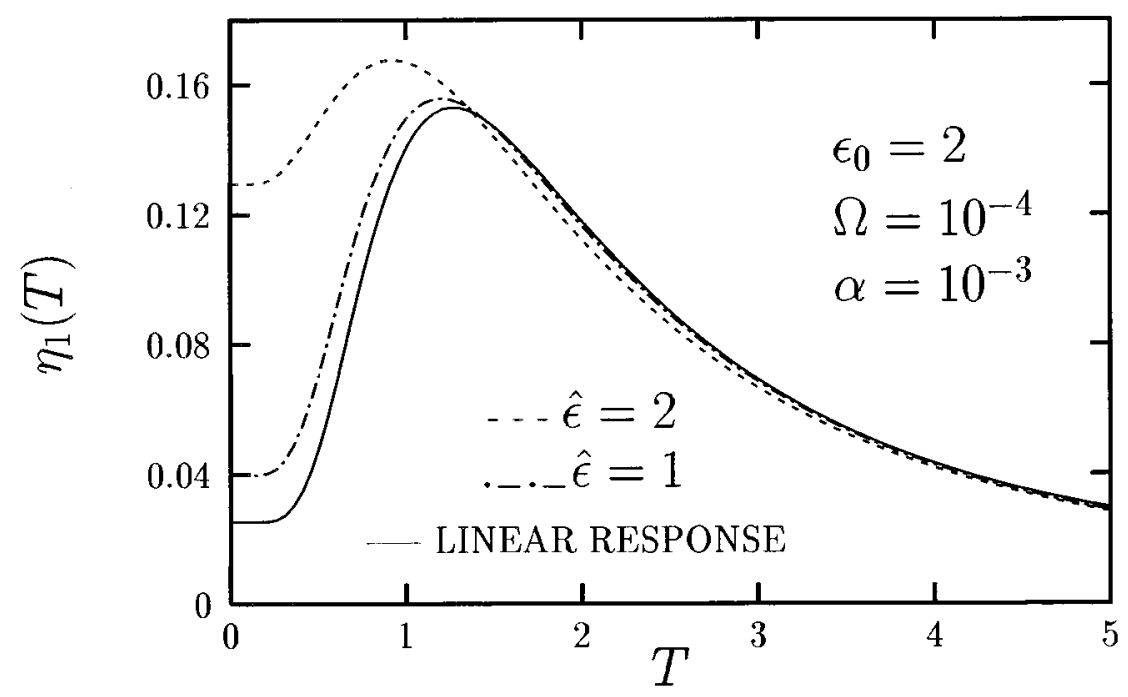

FIG. 8. Amplification vs temperature of the power amplitude $\eta_{1}$ in the adiabatic coherent regime. For intermediate driving strength $\hat{\epsilon}<\epsilon_{0}$ the position and the shape of the maximum are only slightly modified with respect to the linear-response approximation. As the driving strength is increased, the maximum in the nonlinear QSR increasingly disappears. 


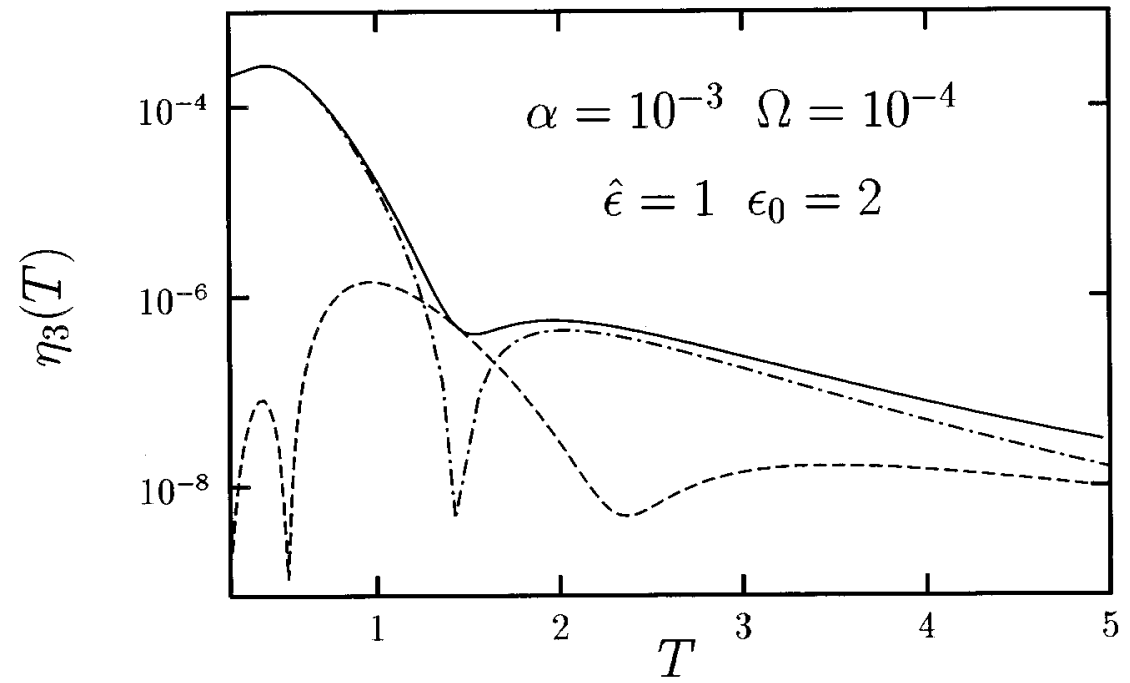

FIG. 9. Noise-induced suppression of the third amplitude $\eta_{3}$ vs temperature in the adiabatic coherent regime ( 5.1) (full line). The dot-dashed and dashed lines depict the quasistatic and the retarded contributions to $\eta_{3}$ [see Eqs. (4.14) and ( 5.2)].

where the retarded contribution vanishes as $\Omega \rightarrow 0$. To be definite, in terms of dimensionless integration variables $x=\Omega t_{0}, y=\Omega t_{1}$, and $z=\Omega t$ one finds from Eq. (4.12) that

$$
P_{m}^{(\mathrm{qs})}(\hat{\boldsymbol{\epsilon}})=\frac{1}{2 \pi} \int_{0}^{2 \pi} d x \tanh \frac{\hbar \varepsilon(x)}{2 k_{B} T} \cos m x
$$

and

$$
\begin{aligned}
P_{m}^{(\mathrm{ret})}(\Omega, \hat{\boldsymbol{\epsilon}})= & \frac{1}{2 \pi} \frac{\hbar \hat{\epsilon}}{2 k_{B} T} \frac{1}{1-e^{-\Gamma}} \int_{0}^{2 \pi} d x \frac{\sin x}{\cosh ^{2}\left[\hbar \varepsilon(x) / 2 k_{B} T\right]} \\
& \times \int_{0}^{2 \pi} d y e^{i m(x+y)} \exp \left(-\int_{0}^{y} d z \gamma_{\alpha}(x+z) / \Omega\right),
\end{aligned}
$$

where $\Gamma=\int_{0}^{2 \pi} d z \gamma_{\alpha}(z) / 2 \pi, z=\Omega t$. Hence the quasistatic contribution is independent of the frequency $\Omega$, while the retarded contribution becomes negligible when $\Omega \ll \gamma_{\alpha}$, which for the parameters chosen in Figs. 6 and 7 amounts to $\Omega \leqslant 10^{-4} \Delta_{e}$. Hence the NIS is most pronounced when theretarded contribution becomes negligible (see also Fig. 9 below).

\section{ADIABATIC QUANTUM COHERENT QSR}

At low temperatures $k_{B} T \leqslant \hbar E$, weak coupling $\alpha \ll 1$, and $\Delta_{e} \geqslant|\varepsilon(t)|$, the NIBA fails to predict the correct long-time behavior because the neglected bath-induced correlations contribute to the dissipative effects to first order in the coupling strength. Nevertheless, at low temperatures, where in the static case quantum coherence occurs (the QC region in the inset of Fig. 1) a perturbative treatment is allowed $[7,8]$. A solution of the dynamics can then be discussed in the low-frequency regime $\hat{\boldsymbol{\epsilon}} \Omega \ll \Delta^{2}$, where the tunneling motion may be treated in the adiabatic limit [25] [region (c) of Fig. 1]. In the adiabatic regime and for weak coupling $P_{\text {as }}(t)$ is simply related to the function $N(t)$, representing the population difference between the lower and upper eigenstates of the driven two-state system, by $P_{\text {as }}(t)=\hbar \varepsilon(t) / E(t) N(t)$. Here $E(t)=\hbar\left[\Delta_{e}^{2}+\varepsilon(t)^{2}\right]^{1 / 2}$ is the time-dependent level splitting. One then finds that $N(t)$ obeys the first-order differential equation $\dot{N}(t)=-\gamma_{\text {rel }}(t)\left[N(t)-N_{\text {eq }}(t)\right]$, which is again in the form of a rate equation with a time-dependent rate $\gamma_{\text {rel }}(t)=\pi \alpha \hbar \Delta_{e}^{2} / E(t) \operatorname{coth} E(t) / 2 k T$ [see Eq. (2.17)] and time-dependent adiabatic equilibrium value $N_{\text {eq }}(t)=\tanh E(t) / 2 k T$. Again this equation can be solved in terms of quadratures to give, for the harmonics of $P_{\text {as }}(t)$, the expression

$$
\begin{aligned}
P_{m}(\Omega, \hat{\boldsymbol{\epsilon}})= & \frac{\Omega}{2 \pi} \int_{0}^{2 \pi / \Omega} d t_{0} \frac{\hbar \varepsilon\left(t_{0}\right)}{E\left(t_{0}\right)} e^{-i m \Omega t_{0}} \\
& \times \int_{0}^{\infty} d t_{1} N_{\mathrm{eq}}\left(t_{1}\right) \gamma_{\mathrm{rel}}\left(t_{1}\right) \\
& \times \exp \left(-\int_{t_{0}}^{t_{0}+t_{1}} d t \gamma_{\mathrm{rel}}(t)\right) .
\end{aligned}
$$

Equations (4.12)-(5.1) capture the essential features of the dynamics of the driven TLS for low frequencies and weak coupling in the whole temperature range. For strong coupling or strong adiabatic asymmetries $|\varepsilon(t)| \gg \Delta_{e}$, Eq. (4.12) holds down to the lowest temperatures. Figure 8 shows the behavior of the power amplification $\eta_{1}$ versus temperature for different external strenghts $\hat{\boldsymbol{\epsilon}}$ compared with the linear-response result (full line). As shown in Fig. 8, the position of the maximum and the shape of the resonance are modified as compared to the linear-response approximation obtained with Eqs. (2.14) and (2.15) (see also Fig. 2). Figure 9 shows the behavior of $\eta_{3}$ versus temperature (full line). Again for adiabatic driving, even in this low-temperature regime, we observe a NIS of higher harmonics as the frequency is decreased. As discussed in Sec. IV [see Eq. (4.14)], we can evaluate any harmonic $\eta_{m}$ as the sum of a quasistatic contribution $\eta^{\text {(qs) }}$ and a retarded contribution $\eta^{\text {(ret) }}$, where the latter vanishes as $\Omega \rightarrow 0$. To be definite, 


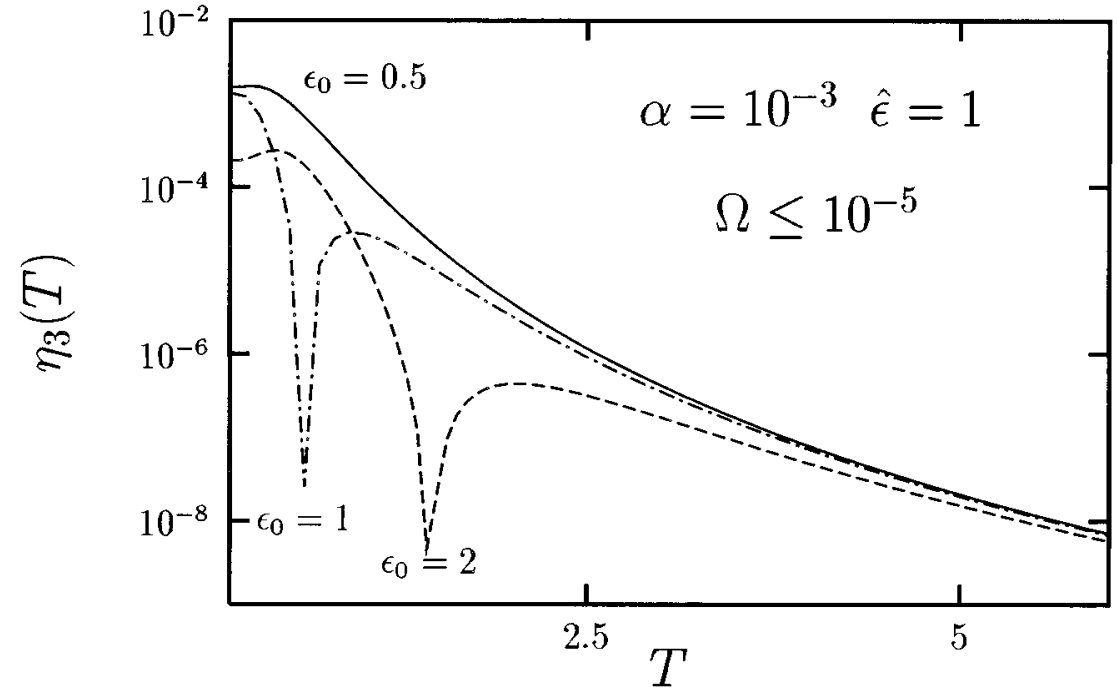

FIG. 10. Noise-induced suppression of the third amplitude $\eta_{3}$ vs temperature in the quasistatic coherent regime [cf. Eq. (5.2)] for different asymmetries $\epsilon_{0}$. The NIS disappears when the external strength $\hat{\epsilon}$ exceeds the intrinsic asymmetry $\epsilon_{0}$.

when the condition $\gamma_{\text {rel }} \ll \Omega$ is fulfilled, Eq. (5.1) may be simplified to the quasistatic expression

$$
P_{m}^{(\mathrm{qs})}=\frac{1}{2 \pi} \int_{0}^{2 \pi} d x \frac{\hbar \varepsilon(x)}{E(x)} \tanh \frac{E(x)}{2 k_{B} T} \cos m x,
$$

which is independent of the external frequency $\Omega$. The dotdashed line of Fig. 9 represents the quasistatic contribution $\eta^{\text {(qs) }}$, while the dashed line is the retarded contribution $\eta^{\text {(ret) }}$, which, for the chosen parameters, is negligible when $\Omega<10^{-5} \Delta_{e}$. Correspondingly (not shown, but see Fig. 6 for the case where the NIBA holds), the phase exhibits a crossover at the very same value of the temperature where the NIS appears. Figure 10 depicts the dependence of the NIS's as the intrinsic asymmetry $\epsilon_{0}$ is varied. It is found that the NIS disappears when the external strength overcomes the intrinsic asymmetry.

\section{CONCLUSIONS}

In summary, we have investigated QSR phenomena in the deep quantum regime. In the nonlinear regime, drivinginduced coherence and quantum coherence may occur. On the other hand, we showed that a maximum in the power amplitude plotted versus temperature (that is, stochastic resonance) occurs only when incoherent tunneling contributions dominate over coherent transitions. Further, we found that quantum noise can substantially enhance, but also suppress, the nonlinear response. In particular, the occurrence of a noise-induced suppression of higher harmonics allows a distortion-free amplification of signals in quantum systems. The possibility of QSR in the presence of coherent tunneling carries a great potential for applications, as they emerge in the task of controlling persistent quantum coherences in complex systems by use of tailored laser pulse sequences [27]. These QSR phenomena may be detected by measuring the ac conductance in mesoscopic metals [5,28], in ac-driven atomic force microscopy [29], investigating ac-driven hydrogen tunneling in metals [30], or in driven macroscopic quantum systems [26]. Hence quantum noise does not represent a nuisance, but rather can be a useful tool when interplaying with external periodic perturbations.

\section{ACKNOWLEDGMENT}

We gratefully acknowledge the support of this work by the Deutsche Forschungsgemeinshaft (Grant No. HA1517/ 14-1).
[1] R. Benzi, A. Sutera, and A. Vulpiani, J. Phys. A 14, L453 (1981).

[2] See, for example, Proceedings of the NATO Workshop on SR in Physics and Biology, edited by F. Moss et al. [J. Stat. Phys. 70, 1 (1993)].

[3] For reviews see P. Jung, Phys. Rep. 234, 175 (1993); F. Moss, D. Pierson, and D. O'Gorman, Int. J. Bif. Chaos 4, 1383 (1994).

[4] M. Grifoni, L. Hartmann, S. Berchtold, and P. Hänggi, Phys. Rev. E 53, 5890 (1996).

[5] R. Löfstedt and S.N. Coppersmith, Phys. Rev. Lett. 72, 1947 (1994); Phys. Rev. E 49, 4821 (1994).

[6] M. Grifoni and P. Hänggi, Phys. Rev. Lett. 76, 1611 (1996).
[7] A.J. Leggett, S. Chakravarty, A.T. Dorsey, M.P.A. Fisher, A. Garg, and W. Zwerger, Rev. Mod. Phys. 59, 1 (1987).

[8] U. Weiss, Quantum Dissipative Systems, Series in Modern Condensed Matter Physics (World Scientific, Singapore, 1993), Vol. 2.

[9] A. Benderskii, D.E. Makarov, and C.A. Wight, Adv. Chem. Phys. 88, 1 (1994).

[10] M. Grifoni, M. Sassetti, J. Stockburger, and U. Weiss, Phys. Rev. E 48, 3497 (1993).

[11] T. Dittrich, B. Oeschlägel, and P. Hänggi, Europhys. Lett. 22, 5 (1993); Acta Phys. Pol. B 94, 845 (1993).

[12] Y. Dakhnovskii, Phys. Rev. B 49, 4649 (1994); Ann. Phys. (N.Y.) 230, 145 (1994); I.A. Goychuk, E.G. Petrov, and V. 
May, Phys. Rev. E 52, 2392 (1995).

[13] M. Grifoni, M. Sassetti, P. Hänggi, and U. Weiss, Phys. Rev. E 52, 3596 (1995).

[14] M. Grifoni, M. Sassetti, and U. Weiss, Phys. Rev. E 53, 2033 (1996).

[15] Numerical studies on the driven symmetric two-state system can be found in D.E. Makarov and N. Makri, Phys. Rev. E 52, 5863 (1995).

[16] B. McNamara and K. Wiesenfeld, Phys. Rev. A 39, 4854 (1989).

[17] R. Bartussek, P. Hänggi, and P. Jung, Phys. Rev. E 49, 3930 (1994); R. Bartussek, P. Jung, and P. Hänggi, in Noise in Physical Systems and 1/f Fluctuations, edited by P.H. Handel and A.L. Chung, AIP Conf. Proc. No. 285 (AIP, New York, 1993), p. 661.

[18] In Fig. 1 we left out the parameter region of low temperatures and nonadiabatic driving where both driving-induced coherence and quantum coherence occur. The dynamics of the driven TLS in this parameter region has been recently investigated in [14].

[19] P. Jung and P. Hänggi, Phys. Rev. A 44, 8032 (1991).

[20] Due to the explicit time dependence of the perturbation, at long times $t+\tau \gg t_{0}, t \gg t_{0}$ (with $t_{0}$ denoting the time of initial preparation), the correlation function $\left\langle\sigma_{z}(t+\tau) \sigma_{z}(t)\right.$ $\left.+\sigma_{z}(t) \sigma_{z}(t+\tau)\right\rangle / 2$ depends separately on the time arguments $t$ and $t+\tau$. After an average over the period, the resulting correlation function $\bar{C}(\tau)$ is again only a function of the time difference $\tau$.

[21] R. Kubo, J. Phys. Soc. Jpn. 12, 570 (1957).

[22] H.B. Callen and T.A. Welton, Phys. Rev. 83, 34 (1951).

[23] U. Weiss and M. Wollensak, Phys. Rev. Lett. 62, 1663 (1989).

[24] M. Sassetti and U. Weiss, Phys. Rev. A, 415383 (1990).

[25] J. Stockburger, M. Grifoni, and M. Sassetti, Phys. Rev. B 51, 2835 (1995).

[26] R. Rouse, S. Han, and J.E. Lukens, Appl. Phys. Lett. 66, 108 (1995); A.D. Hibbs, A.L. Singaas, E.W. Jacobs, A.R. Bulsara, J.J. Bekkedahl, and F. Moss, J. Appl. Phys. 77, 2582 (1995).

[27] D.J. Tannor, Nature 369, 445 (1994); P. Brumer and M. Shapiro, Annu. Rev. Phys. Chem. 43, 257 (1992).

[28] B. Golding, N.M. Zimmerman, and S.N. Coppersmith, Phys. Rev. Lett. 68, 998 (1992); K. Chun and N.O. Birge, Phys. Rev. B 48, 11500 (1993).

[29] D.M. Eigler and E.K. Schweitzer, Nature 344, 524 (1990). Here the quantum dissipation for Xe atoms tunneling between an atomic force microscope tip and a $\mathrm{Ni}(100)$ surface is Ohmic-like and quantum coherent tunneling has indeed been observed experimentally [E.K. Schweitzer (private communication)].

[30] Quantum coherent oscillations with Ohmic coupling have been observed for hydrogen tunneling in niobium by H. Wipf et al., Europhys. Lett. 4, 1379 (1987). 